\title{
Nanophotonics based on Planar Photonic Crystals
}

\author{
Marko Loncar, Tomoyuki Yoshie, Jelena Vuckovic and Axel Scherer \\ California Institute of Technology, MS 136-93, Pasadena, CA 91125
}

Hao Chen and Dennis Deppe, University of Texas, Austin, TX 78712-1084

Pawan Gogna and Yueming Qiu

Jet Propulsion Laboratory, California Institute of Technology, MS 302-306, Pasadena, CA 91109

Dusan Nedeljkovic and Thomas P. Pearsall,

Centre Européen de Recherche de Fontainebleau, Corning, SA 77210 Avon, France

e-mail: loncar@caltech.edu, phone: (626) 395-2207, fax: (626) 683-9547, http://nanofab.caltech.edu

Planar photonic crystals (PPC) are a recent innovation that can permit the miniaturization of optical devices to a scale comparable to the wavelength of light, and their integration in large numbers in the same way as electronic components have been integrated to form microchips. These periodic structures can be designed to open up frequency bands within which the propagation of electromagnetic waves is forbidden irrespective of the propagation

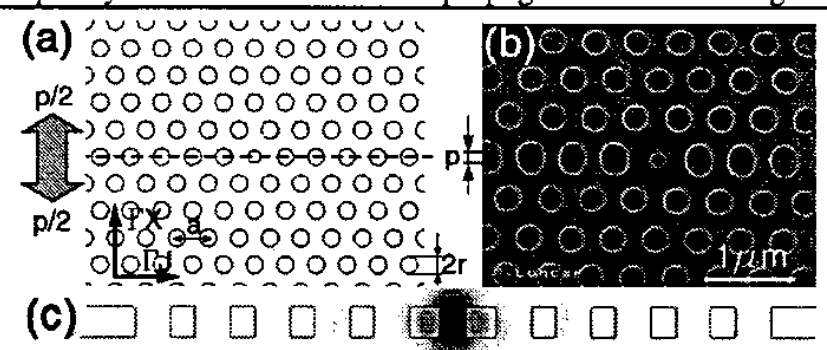

Fig. 1. (a) Schematic of conventional cavity and (b) SEM micrograph of fabricated high- $Q$ cavity. (c) Optical field intensity in the case of high- $Q$ cavity (cross-section), result of 3D FDTD. direction. By creating different types of defects in the photonic crystal lattice, various nanophotonics components, such as cavities and waveguides, can be realized. The quest for a compact and efficient nano-cavity, with high quality factor (Q) and small mode volume $\left(V_{\text {mode }}\right)$, has been a central part of research in the field of integrated optics. Recently, ${ }^{1}$ we have proposed a systematic method to design optical nano-cavities that satisfy both of these requirements. The cavity consists of a defect hole that is smaller than surrounding holes arranged in the triangular lattice photonic crystal. The row that contains the defect hole is elongated by moving two PPC half-planes a fraction of a lattice constant apart in the $\Gamma \mathrm{X}$ direction, with a dislocation parameter $p$ (Fig. 1). We have shown that by tuning $p$ parameter $Q$ factors of single defect cavities can be significantly improved $(Q>10,000)$, while maintaining a small mode volume $\left[\mathrm{V}_{\text {mode }} \approx 0.1(\lambda / 2)^{3}\right]$. The cavity is designed for cavity $\mathrm{QED}$ experiments where strong coupling between photons trapped in the cavity and atoms placed in the small hole in the center of the cavity (where the optical field intensity is the strongest) is to be investigated [Fig. 1(c)].

In order to test our design in the experiment, we have fabricated high- $Q$ cavities in InGaAsP material system. Optical gain is provided by four $9 \mathrm{~nm}$ thick, compressively strained, quantum wells with an electronic bandgap at $\mathrm{E}_{\mathrm{g}}=1.55 \mu \mathrm{m}$, positioned in the center of $330 \mathrm{~nm}$ thick InGaAsP slab $\left(\mathrm{E}_{\mathrm{g}}=1.22 \mu \mathrm{m}\right)$. The slab is positioned on the top of $1 \mu \mathrm{m}$ thick sacrificial InP layer, followed by an InGaAs etch stop and InP substrate. Fabrication
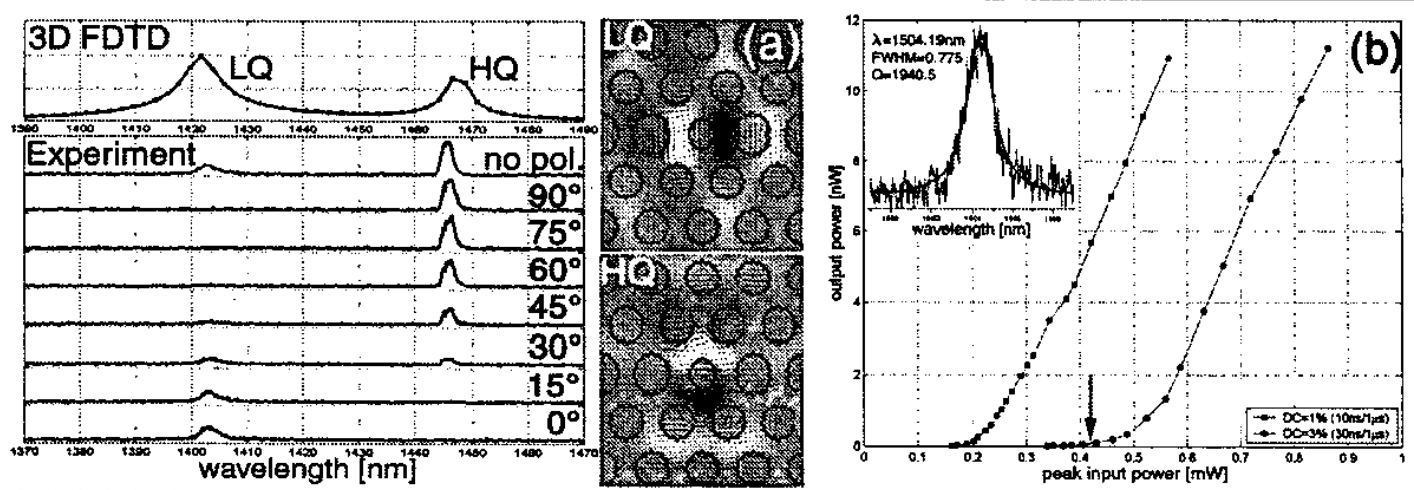

Fig. 2. (a) Polarization dependence of the two modes detected in the $p / a=15 \%$ cavity. Mode profiles $\left(B_{z}\right)$ and polarization ( $E$ field) of the two modes are also shown. (b) L-L curve for HQ mode in $p / a=25 \%$ cavity, taken at two different duty cycles (DC). Spectrum taken below threshold (arrow) is shown in the inset. 
procedure can be found elswhere ${ }^{2}$. The final structure is a suspended membrane, perforated with a $2 \mathrm{D}$ triangular lattice of holes $(\mathrm{a}=435 \mathrm{~nm})$ within which a high-Q cavity is defined.

The structures were tested using micro-photoluminescence approach, and were optically pumped with 1030ns long pump pulses (periodicity $1 \mu \mathrm{s}$ ). The emission from the cavities is collected through the objective lens, and the spectrum of the emitted light signal is analyzed with an optical spectrum analyzer. Using this technique, we have found that high-Q cavity supports two linearly polarized resonances that have orthogonal polarization [Fig. 2(a)]. This result is in an excellent agreement with our 3D FDTD analysis. We have also found that the mode at longer wavelengths $(\mathrm{HQ})$ has much higher $\mathrm{Q}$-factor values $(\mathrm{Q} \approx 2,000)$, and that the position of the two resonances depends strongly on the value of the elongation parameter $p$, as predicted in our earlier publication ${ }^{1}$. Room temperature lasing $^{2}$, with the lowest threshold pumping powers reported for photonic crystal laser (below $220 \mu \mathrm{W}$ ), was observed from the HQ mode [Fig. 2(b)]. The mode profiles taken by our IR camera show that the lasing resonance is well localized to the center of the cavity. Interesting feature of our laser design is presence of the air hole at the center of the structure, at the position of maximum field intensity. Therefore the structure can be used for chemical sensing and nanospectroscopy. In spite of this unusual design, and reduced overlap with the gain region, we observe record-low threshold powers in our devices. We have attributed this to the small mode volume and the high $\mathrm{Q}$ factors inherent to our device design.

We have also fabricated similar high-Q cavity structures in $\mathrm{Al}_{0.16} \mathrm{Ga}_{0.84}$ As slab (thickness 240nm) that contains three InAs quantum dot layers ${ }^{3}$. Final structure is again suspended photonic crystal membrane, with lattice spacing $a=370 \mathrm{~nm}$. Fig. 3 shows dependence of photoluminescence spectrum on the dislocation parameter $p / a$. The measured $\mathrm{Q}$ was as high as 2,800 , what is the record Qfactor value reported so far in the case of photonic crystal cavity. To compare the measured Q with our simulation results, we have carefully measured the geometries of our fabricated structures, and modeled these with 3D-FDTD method. $Q$ value calculated in this case $(Q=4,400)$ is in good agreement with experimentally estimated value. Polarization properties of experimentally measured HQ and LQ modes are also found to be in a very good agreement with theoretical 3D FDTD predictions.

We have shown above that it is possible to generate light and confine it efficiently with high-Q optical nanocavities. However, if a nanophotonics system is to be constructed, it is also necessary to guide light from one device to another ${ }^{4,5}$. Therefore, we have conducted extensive tests on photonic crystal waveguides to explore the opportunities of using this geometry to efficiently guide light, especially around very sharp bends [Fig. 4(c)]. We have observed evidence of guiding in these structures ${ }^{6}$ and we have been able to experimentally obtain the dispersion diagram of guided modes $^{7}$ [Fig. 4(b)]. We have also proposed novel methods to control dispersion properties of these guided modes ${ }^{8}$.

1. J. Vuckovic et al., Phys. Rev. E, 65, 016608 (2001).

2. M. Loncar et al., submitted to Appl. Phys. Lett. June 2002.

3. T. Yoshie et al., $A P L$., 79, 4289 (2001)

4. T. Baba et al., Elect. Lett. 37,761 (2001).

5. M. Notomi et al., Elect. Lett. 37, 293 (2001).

6. M. Loncar et al., $A P L, 77,1937(2000)$.

7. M. Loncar et al., $A P L, 80,1689$ (2002).

8. M. Loncar et al., JOSA $B, 18,1362$ (2001).

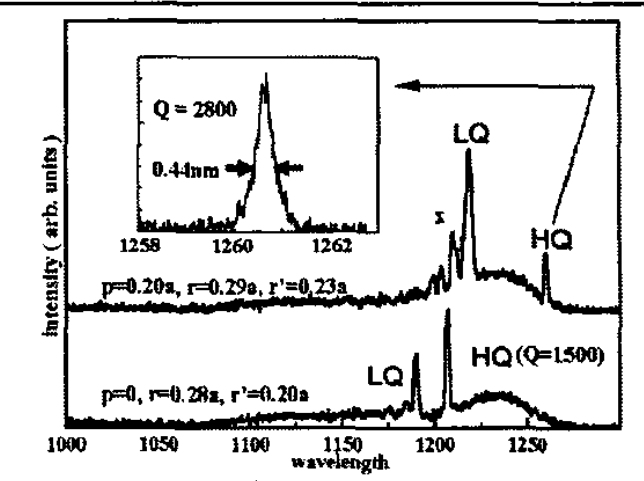

Fig. 3. PL spectrum of structures with different $p / a$
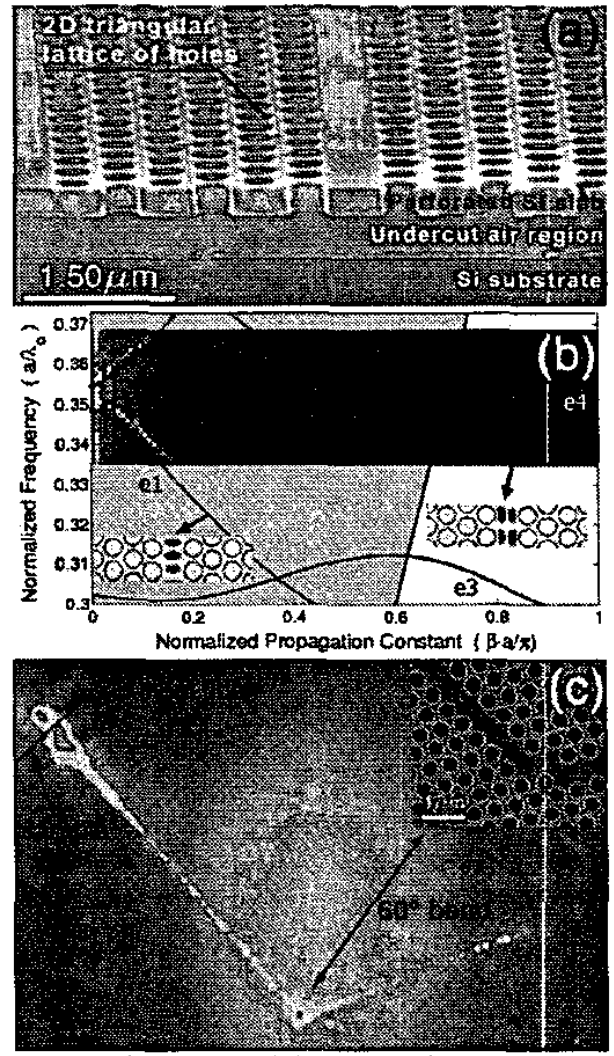

Fig. 4. (a) Photonic crystal single line defect waveguide and its (b) dispersion diagram: solid lines - theory, white pixels - experiment. (c) Guiding around sharp $60^{\circ}$ bend. 